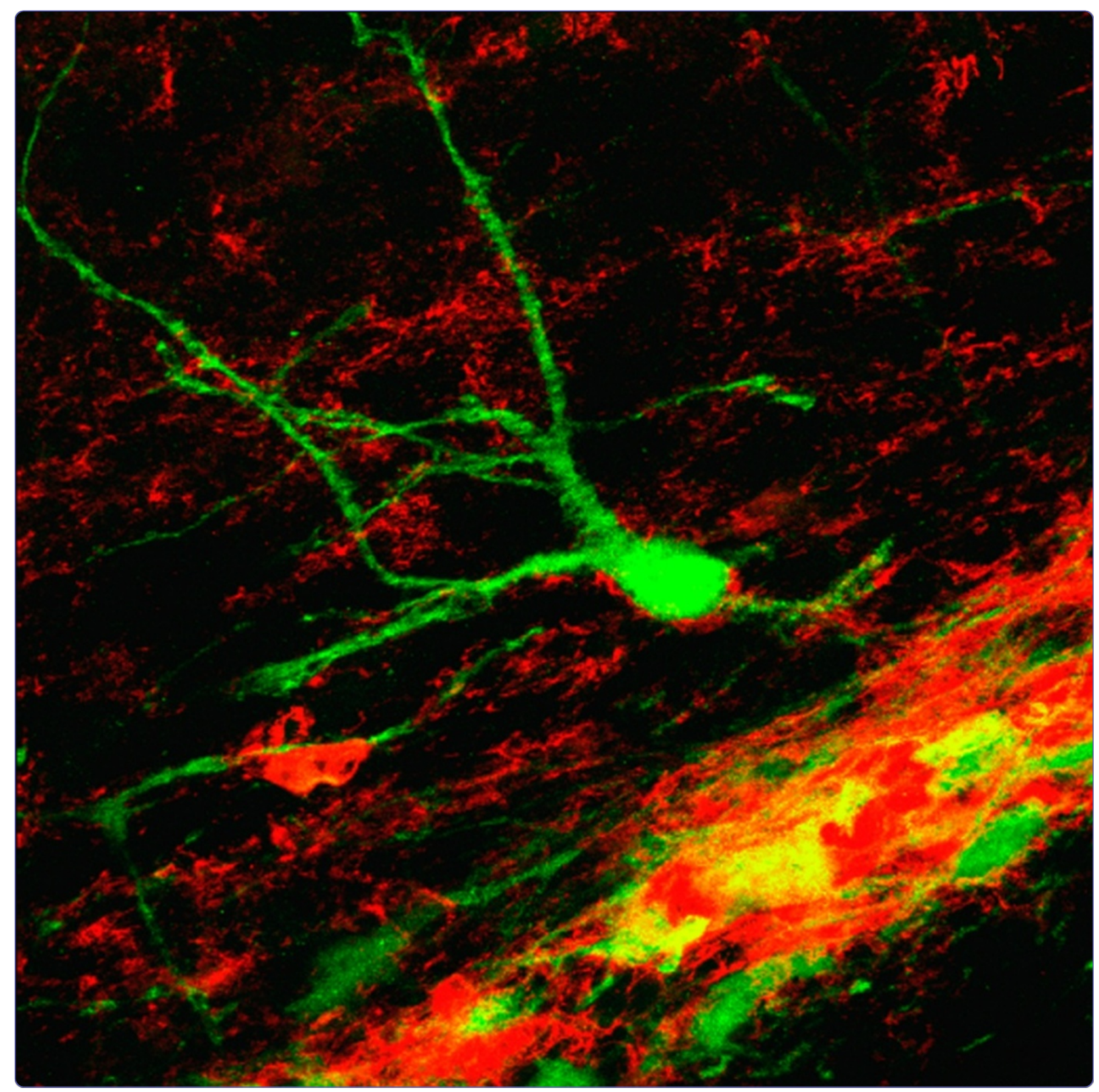

\title{
Neural stem cells genetically-modified to express neprilysin reduce pathology in Alzheimer transgenic models
}

Blurton-Jones et al. 


\title{
Neural stem cells genetically-modified to express neprilysin reduce pathology in Alzheimer transgenic models
}

\author{
Mathew Blurton-Jones ${ }^{1 *}$, Brian Spencer ${ }^{4}$, Sara Michael ${ }^{4}$, Nicholas A Castello ${ }^{1}$, Andranik A Agazaryan ${ }^{1}$, Joy L Davis ${ }^{1}$,
} Franz-Josef Müller ${ }^{2,3}$, Jeanne F Loring ${ }^{2}$, Eliezer Masliah ${ }^{4}$ and Frank M LaFerla ${ }^{1}$

\begin{abstract}
Introduction: Short-term neural stem cell (NSC) transplantation improves cognition in Alzheimer's disease (AD) transgenic mice by enhancing endogenous synaptic connectivity. However, this approach has no effect on the underlying beta-amyloid $(A \beta)$ and neurofibrillary tangle pathology. Long term efficacy of cell based approaches may therefore require combinatorial approaches.

Methods: To begin to examine this question we genetically-modified NSCs to stably express and secrete the $A \beta$-degrading enzyme, neprilysin (sNEP). Next, we studied the effects of sNEP expression in vitro by quantifying A $\beta$-degrading activity, NSC multipotency markers, and A $\beta$-induced toxicity. To determine whether sNEP-expressing NSCs can also modulate AD-pathogenesis in vivo, control-modified and sNEP-NSCs were transplanted unilaterally into the hippocampus of two independent and well characterized transgenic models of AD: 3XTg-AD and Thy 1-APP mice. After three months, stem cell engraftment, neprilysin expression, and AD pathology were examined.

Results: Our findings reveal that stem cell-mediated delivery of NEP provides marked and significant reductions in $A \beta$ pathology and increases synaptic density in both 3xTg-AD and Thy 1-APP transgenic mice. Remarkably, A $\beta$ plaque loads are reduced not only in the hippocampus and subiculum adjacent to engrafted NSCs, but also within the amygdala and medial septum, areas that receive afferent projections from the engrafted region.

Conclusions: Taken together, our data suggest that genetically-modified NSCs could provide a powerful combinatorial approach to not only enhance synaptic plasticity but to also target and modify underlying Alzheimer's disease pathology.
\end{abstract}

\section{Introduction}

Alzheimer's disease (AD) is the leading cause of agerelated dementia, afflicting one in every eight people over the age of 65 [1]. Unfortunately, the incidence of dementia is expected to quadruple over the next 40 years, such that more than 115 million people will be afflicted world-wide by 2050 [2]. Currently approved therapies offer only marginal, temporary relief and fail to modify the underlying disease pathology. Thus, there is a critical and urgent need to examine novel and combinatorial approaches to treat this devastating disorder.

\footnotetext{
* Correspondence: mblurton@uci.edu

'Department of Neurobiology and Behavior and Institute for Memory Impairment and Neurological Disorders, University of California Irvine, Irvine, CA 92697, USA

Full list of author information is available at the end of the article
}

Pathologically, $\mathrm{AD}$ is characterized by the accumulation of two hallmark brain lesions, beta-amyloid (A $\beta)$ plaques and neurofibrillary tangles (NFTs). A $\beta$ plaques result from the extracellular accumulation of insoluble aggregates of a small 40 to 42 amino acid peptide, A $\beta$. NFTs in contrast, consist of intraneuronal insoluble aggregates of the microtubule binding protein, tau, which becomes hyperphosphorylated and compromises neuronal activity, connectivity and function. In addition to the accumulation of $A \beta$ and tau across multiple brain regions, $\mathrm{AD}$ patients also exhibit inflammation and widespread synaptic and neuronal loss.

Given the complex nature of this disease and the multiple pathways and regions affected, a single small molecule approach may not provide substantial benefit in the absence of other interventions. Biologic-based approaches, such as stem cell transplantation, are therefore 
receiving increasing attention. Recently, we and others showed that neural stem cell (NSC) transplantation markedly improves cognitive function, synaptic connectivity and neuronal survival in models of AD and tauopathy [3,4]. Many of these effects appear to be mediated by stem cellderived neurotrophins or other neuroprotective activities. Mesenchymal stem cells have also been found to improve cognition in $\mathrm{AD}$ models by modulating cytokine levels and brain inflammation [5]. Thus, stem cells provide therapeutic efficacy in preclinical models of $\mathrm{AD}$ by modulating complex biological systems via multiple mechanisms. Although short-term benefits of stem cell transplantation appear promising and warrant further examination, these studies have also shown that NSCs do not modify the underlying $A \beta$ or tau pathology $[3,4]$. Hence, it is critical to examine combinatorial approaches aimed at not only improving synaptic connectivity and neuronal function but also diminishing $A \beta$ and tau accumulation.

Over the last decade, pathways that underlie the endogenous degradation of $A \beta$ have been identified. Among these, the proteolytic enzyme neprilysin (NEP) exhibits some of the most potent activity [6,7]. Interestingly, levels and activity of NEP are decreased in AD brains, suggesting that a reduction in $A \beta$ degradation may contribute to the development of the disease $[8,9]$. Approaches that enhance or harness these degradation pathways may provide important disease-modifying efficacy. In support of this, transgenic or viral-mediated overexpression of NEP reduces A $\beta$ pathology $[7,10]$. Unfortunately, widespread delivery of these therapeutic proteins to the large human brain will likely be required, but is hindered by the blood brain barrier and the limited radius of infectivity $(<0.5 \mathrm{~mm})$ afforded by current viral-based approaches [11]. One possible solution to this problem is to harness the migratory activity of NSCs [12] and use these cells to deliver $A \beta$-degrading enzymes throughout the forebrain.

In this study, we hypothesized that NSCs could provide an effective means to deliver disease-modifying therapeutic proteins to the brain. To test this hypothesis, we generated murine NSC lines that overexpress a secreted form of the $\mathrm{A} \beta$-degrading enzyme, neprilysin (sNEP). In vitro characterization of these cells revealed high levels of sNEP protein expression and no changes to NSCs markers or differentiation potential. sNEP expression also protected NSCs against A $\beta$-induced toxicity both in vitro and in vivo. Most critically, these cells survive and continue to produce sNEP for several months following transplantation, markedly reducing $A \beta$ pathology and enhancing synaptic connectivity in two independent transgenic models. Thus, sNEP-expressing NSCs represent a promising therapeutic approach that combines the neurotrophic-mediated benefits of stem cell transplantation with the widespread delivery of a disease-modifying protein.

\section{Methods}

Mice

All animal experiments were approved by the University of California, Irvine Institutional Animal Care \& Use Committee and were performed in strict accordance with National Institutes of Health guidelines. The $3 x T g-A D$ and Thy1-APP mice have previously been described [13-15]. 3xTg-AD mice were generated by comicroinjecting transgenes expressing human $\mathrm{APP}_{695}$ with the Swedish mutation (KM670/671NL) and human tau with the P301L mutation under control of the murine Thy1.2 promoter into single-cell embryos of homozygous $P S 1_{M 146 V}$ knockin mice. Thy1-APP mice were generated by pronuclear injection of a transgene expressing $\mathrm{APP}_{751}$ with Swedish and London (V717I) mutations under control of the murine Thy1 promoter. The $3 x \mathrm{Tg}$ AD mice are maintained on a hybrid C57BL6/129 background and Thy1-APP mice are maintained as purebred C57/B16. Equivalent numbers of both male and female mice were utilized in this study and housed on a 12-hour light/dark schedule with ad libitum access to food and water. Importantly, both C57Bl6 and 129 background strains have the identical major histocompatibility complex (MHC) haplotype (H-2b) as the C57Bl6-derived NSCs utilized in this study.

\section{Neural stem cells}

The neural stem cells used for transplantation into 3xTg-AD mice were isolated from postnatal day 1 GFPexpressing transgenic mice as previously described [16]. NSCs transplanted into Thy1-APP mice were derived from the C57Bl6 E15 cortex (Millipore SCR029). NSC lines were grown as adherent monolayers using standard NSC media ((D)MEM/F12 with Glutamax, N2, epidermal growth factor (EGF)). To generate lines that stably expressed s-NEP or empty vector control (pBOBI), GFPNSC cultures were transfected with $5 \mu \mathrm{g}$ of plasmid using an AMAXA nucleofector (program A-33) following standard protocols (Lonza Inc. Alpharetta, GA, USA). Cells were maintained using standard media and three days after nucleofection, selective antibiotic (Zeocin $100 \mathrm{ng} / \mathrm{ml}$, Invivogen, San Diego, California, USA) was added. Media were changed every two days and cells were split as needed. After six weeks of stable selection, cells were frozen down or utilized for experiments. For Thy1-APP studies, NSCs were transduced with lentiviral particles (multiplicity of infection $(\mathrm{MOI})=50$ ) generated by transfection of HEK293 cells with s-NEP or p-BOBI lentiviral and packaging plasmids as described [10]. For transplantation, control and s-NEP-expressing NSCs were lightly trypsinized, washed three times, triturated and filtered through a $70 \mu \mathrm{m}$ mesh. Cells were then counted and resuspended in vehicle $(1 \times$ Hanks balanced salt solution with $20 \mathrm{ng} / \mathrm{ml} \mathrm{hEGF)} \mathrm{at} \mathrm{a} \mathrm{density} \mathrm{of} 50,000$ cells $/ \mu$ l. 


\section{Stereotactic surgeries}

Control- and sNEP-transfected NSCs were delivered to the hippocampus or subiculum using a stereotaxic apparatus. For $3 \mathrm{xTg}-\mathrm{AD}$ mice, NSCs were injected into the subiculum using the following coordinates relative to Bregma: AP: -3.6, ML: \pm 2.1 , DV: -2.0 . Thy1-APP mice received intrahippocampal injections using coordinates: AP: -2.0 , ML: \pm 1.5 , DV: -1.3 . The 3xTg-AD mice were anesthetized with isofluorane, placed in the stereotax and injected with 100,000 control-transfected NSCs into one subiculum/ hippocampus $(2 \mu \mathrm{l} /$ injection) and 100,000 sNEP-NSCs into the other hippocampus using a $5 \mu$ l Hamilton microsyringe (30-gauge) and an injection rate of $0.5 \mu \mathrm{l} /$ minute. The side receiving SNEP cells was randomly chosen and recorded for each animal. Thy1-APP mice underwent similar surgeries except that one side of the hippocampus received a vehicle injection whereas the other side received either sNEP- or control-NSCs. Accurate placement of the injection to the targeted region was confirmed for all animals by visualization of the needle tract within coronal brain sections.

\section{Tissue processing}

The $3 x \operatorname{Tg}-\mathrm{AD}$ mice were sacrificed three months after transplantation and the Thy1-APP mice were sacrificed one month after transplantation via Nembutal overdose and cardiac perfusion with $0.01 \mathrm{M}$ phosphate-buffered saline (PBS) followed by $4 \%$ paraformaldehyde ( $\mathrm{pH}$ 7.4). Brains were rapidly removed and post-fixed for an additional 24 hours at $4^{\circ} \mathrm{C}$. Prior to sectioning, a notch was made in the bottom left cortex so that left versus right sides could be readily distinguished after sectioning. Brains were then cut in $50 \mu \mathrm{m}$ thick coronal sections on a Vibratome and stored in PBS with $0.02 \% \mathrm{NaN}_{3}$ at $4^{\circ} \mathrm{C}$ until use.

\section{Immunofluorescent labeling}

Fluorescent labelling followed standard protocols as previously described $[3,17]$. Primary antibodies utilized include: antibodies against A 3 : 6E10 (Signet, Dedham, MA, USA), fibrillar A $\beta$ (OC, gift of C. Glabe), Tau: HT7 (Innogenetics, Alpharetta, GA, USA), phospho-Tau (Ser 199/202, AT8 epitope; Invitrogen, Carlsbad, CA, USA), glial fibrillary acidic protein (GFAP) (Dako, Carpinteria, CA, USA), $\beta$-III-tubulin, GFP, GalC, active caspase- 3 and CNPase (Millipore, Carlsbad, CA, USA), Doublecortin (Santa Cruz Biotech, Santa Cruz, CA, USA), and synaptophysin (Sigma, St. Louis, MO, USA). Primary antibodies were applied overnight at $4^{\circ} \mathrm{C}$ and detected with appropriate Alexa Fluor conjugated secondary antibodies (Invitrogen). Specificity of all primary antibodies was confirmed by Western blot and by omission of primary antibody in immunofluorescent labelling (data not shown).

\section{Biochemical examination}

SDS-PAGE western blots and $A \beta$ sandwich ELISAs were performed following standard protocols as previously described [3]. Primary antibodies used for western blot analysis include: nestin, sox-2, musashi-1 (Millipore), NEP/CD10 (Vector Biolabs, Burlingame, CA, USA), and brain-derived neurotrophic factor (BDNF, Santa Cruz Biotechnology). To test the effects of $A \beta$ treatment on NSC viability cells were treated with $10 \mathrm{nM} \mathrm{A} \beta 42$ for 24 hours and then lactate dehydrogenase (LDH) release into the media was measured using the CytoTox 96 LDH assay (Promega Corporation, Madison, WI, USA) following the manufacturer's protocol.

\section{Confocal microscopy and quantification}

Immunofluorescent sections were visualized using a Leica TCS SPE or Biorad 1024 Confocal microscope. To avoid non-specific bleed-through, each laser line was excited and detected independently. All images represent either single confocal Z-slices or Z-stacks. For quantitative analysis, slides were coded and then images captured by a blinded observer using identical laser and detection settings. Grayscale Z-stack images were then analyzed using Image J software. Three square regions of interest (ROIs) were randomly defined within the area to be quantified (for example, subiculum) and then mean pixel intensity was computed by the software for each ROI. The three fields were averaged for each sample and means were compared by paired $\mathrm{t}$-test.

\section{Statistical analysis}

Comparisons between multiple groups were performed using analysis of variance (ANOVA) followed by Fischer's PLSD post hoc tests. Groups were considered significantly different when $P<0.05$ for both the ANOVA and post hoc comparisons. $A \beta$ measurements were quantified from opposing sides of the brain from the same individual animals; the data were therefore compared by paired t-test. All statistical analysis was performed using Graphpad Prism software.

\section{Results}

sNEP-expressing neural stem cells remain multipotent, degrade $A \beta$ in vitro, and are resistant to $A \beta$ toxicity

To determine whether NSCs can be genetically modified to promote $A \beta$ degradation, we stably transfected murine GFP-NSCs with a plasmid encoding sNEP or an empty vector as a control. In parallel, we generated a second line of sNEP-expressing NSCs via lentiviral transduction. sNEP expression and multipotency markers were compared by western blot and immunocytochemistry. As shown, sNEPexpressing NSCs produce over 25 fold higher levels of NEP than control transfected NSCs, which express only low levels of full-length NEP (Figure 1A, Figure 2A,B). To determine whether SNEP expression in any way alters NSC multipotency, we examined three well-established NSC markers, sox-2, nestin, and musashi- 1 . Western blot analysis confirmed that sNEP- and control-transfected 

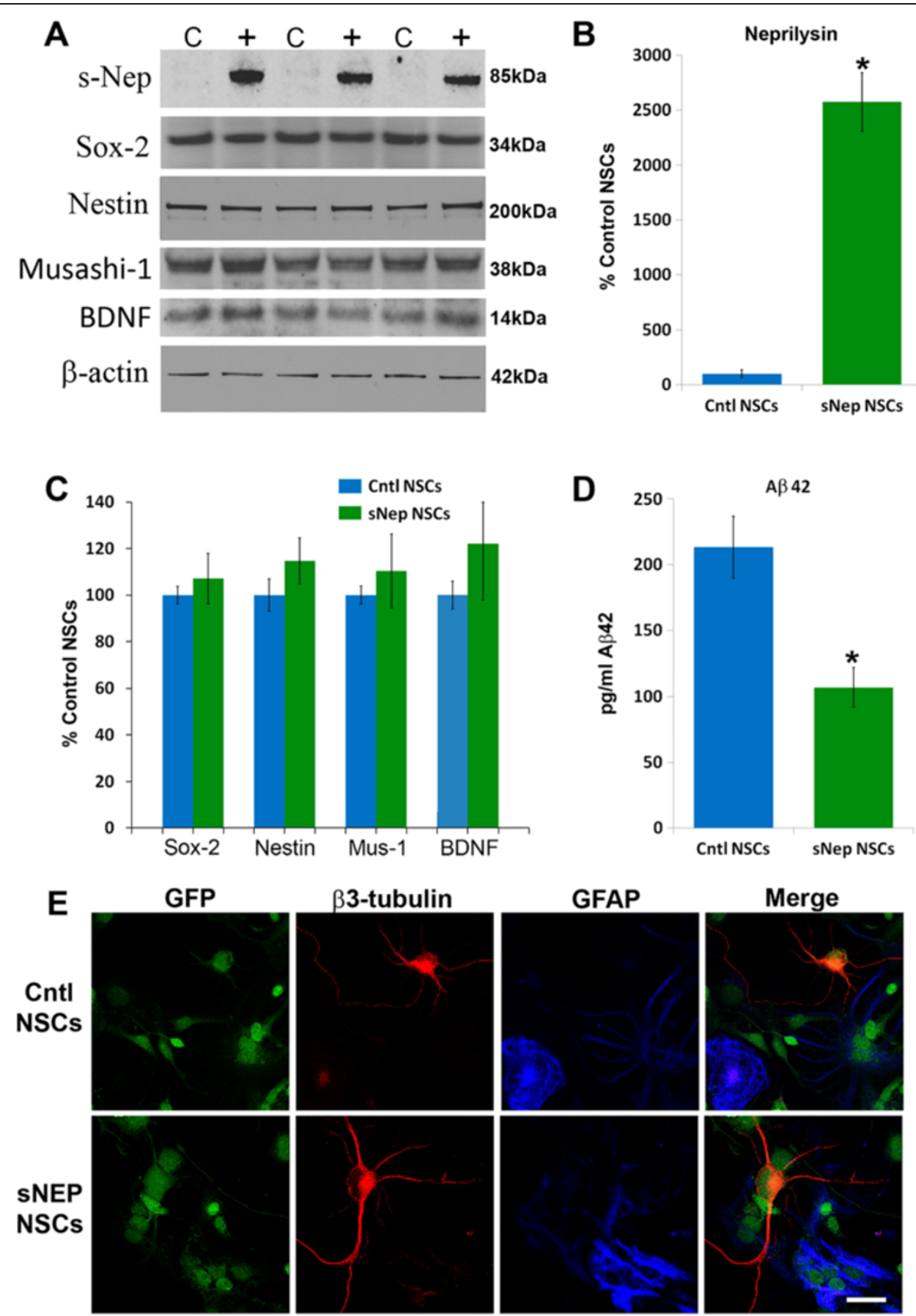

Figure 1 Stably-transfected sNEP-NSCs remain multipotent and efficiently degrade $\mathbf{A} \boldsymbol{\beta}$. (A) Western blot analysis of control (C) and sNEP $(+)$ stably-transfected NSCS (+) reveal high levels of sNEP expression versus control transfected NSCs (quantified in B). Notably, sNEP expression has no effect on markers of NSC multipotency (sox-2, nestin and musashi-1) and does not alter NSC BDNF expression (quantified in C). To determine whether sNEP-expressing cells can degrade $A \beta$, cultures were treated with recombinant human $A \beta 42$ peptide and 48 hours later, conditioned media were collected and A $\beta$ levels measured by sandwich ELISA. (D) sNEP-expressing NSCs efficiently reduced A $\beta$ levels within conditioned media by more than 50\% ( $P=0.0008)$. (E) When NSCs are cultured without mitogen, both control and sNEP-expressing NSCs differentiate into neurons (red, $\beta 3$-tubulin) and glia (blue, GFAP). $N=10$ wells/group, error bars represent standard error of the mean (SEM). Scale Bar $=20 \mu \mathrm{m}$. A $\beta$, beta-amyloid; BDNF, brain-derived neurotrophic factor; GFAP, glial fibrillary acidic protein; NSCs, neural stem cells; SNEP, secreted neprilysin.

NSCs express equivalent levels of these multipotency markers (Figure 1A,C). Likewise, immunocytochemistry confirmed equivalent expression of the intermediate filament protein, nestin within virally-transduced sNEP and control NSCs (Figure 2D,E). We previously showed that NSCs can improve cognition in AD transgenic mice by a mechanism involving BDNF [3]. Hence, we examined BDNF expression by western blot and verified that levels 


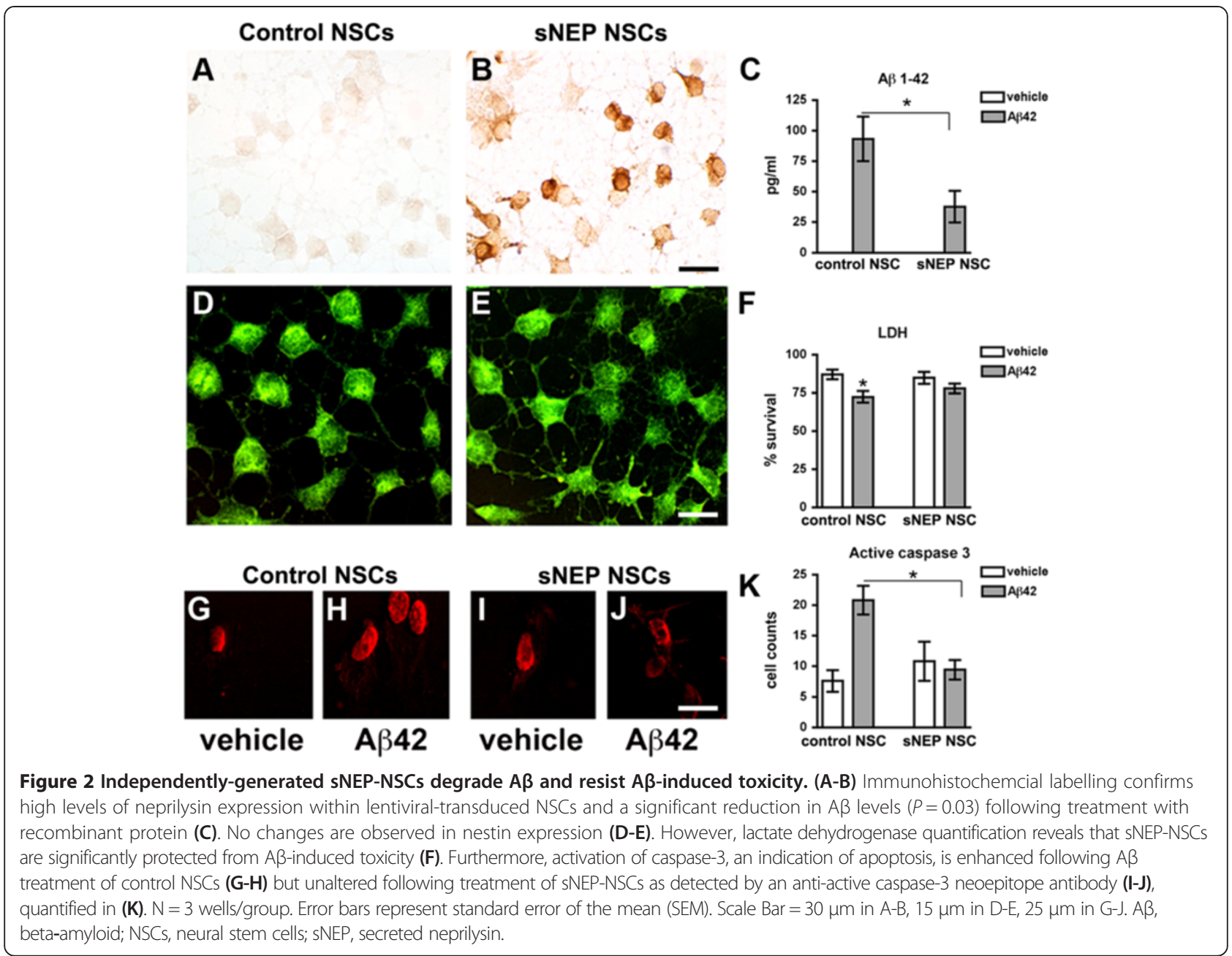

of this important protein were also unchanged (Figure 1A, C). To further confirm that SNEP-expressing NSCs remain multipotent, cells were allowed to differentiate spontaneously by removing EGF from the culture medium and growing cells in neurobasal media supplemented with B27. One week later, cultures were examined by immunofluoresence, confirming equivalent neural and glial differentiation of sNEP- and control-transfected NSCs (Figure 1E).

To determine whether sNEP-expressing NSCs can efficiently degrade $A \beta$, cultures of control- and sNEPexpressing NSCs were treated with recombinant human $\mathrm{A} \beta 42$ peptide and, 48 hours later, $\mathrm{A} \beta$ levels remaining within the media were measured by ELISA. Both the stably-transfected and lentiviral-transduced NSC lines efficiently degraded $A \beta$ in vitro, reducing $A \beta$ levels by more than $50 \%$ (Figure 1D, $P=0.0008$ and Figure $2 C, P=0.03$ ). Interestingly, we found that treatment of NSCs with 10 nM A 342 can trigger caspase-mediated cell death of NSCs within 24 hours (Figure 2). However, sNEP-expression significantly protects NSCs as evidenced by decreased
LDH release and reduced caspase-3 activation versus A $\beta$-treated control NSCs $(P=0.03)$.

\section{Transplanted sNEP-NSCs secrete neprilysin and degrade $A \beta$ in vivo}

Having verified that sNEP expression can promote $A \beta$ degradation in vitro, we next performed stereotactic surgery to deliver sNEP-NSCs and control-NSCs into the brains of transgenic AD mice. NSCs were targeted to the subiculum or hippocampus of AD transgenic mice as both these regions develop robust $A \beta$ plaque pathology, exhibit significant synaptic degeneration and are critical for learning and memory. Importantly, the hippocampus and subiculum are also dramatically affected in AD patients. Two complementary paradigms were pursued to deliver NSCs. In one set of experiments, 18-month-old 3xTg-AD transgenic mice were transplanted unilaterally with 100,000 sNEP-expressing NSCs into the subiculum and 100,000 control-transfected NSCs into the contralateral subiculum. Three months later, the 3xTg-AD mice 

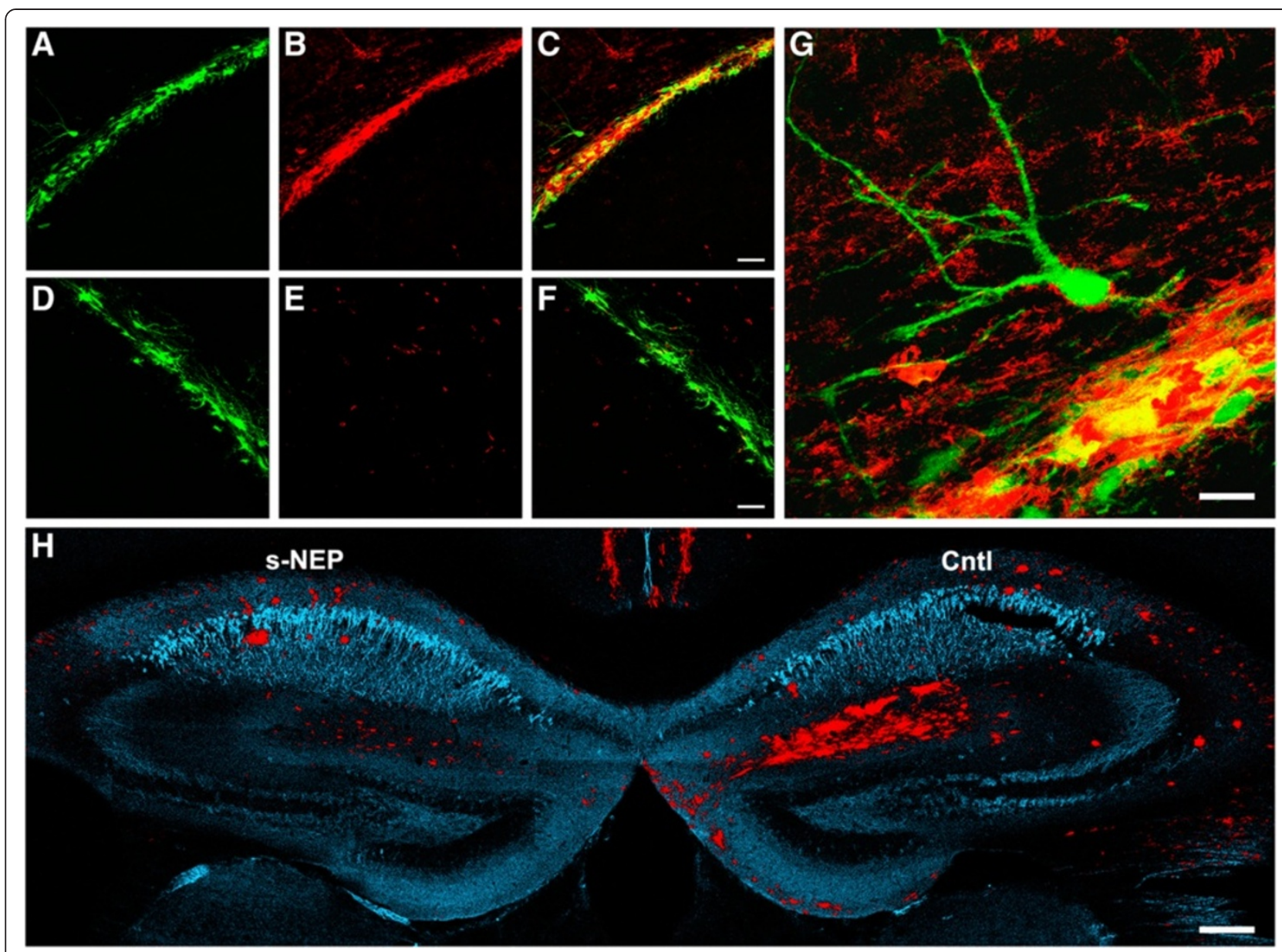

Figure 3 Genetically-modified NSCs continue to produce neprilysin three months after unilateral transplantation and decrease $A \beta$ load within the ipsilateral hippocampus of aged 3xTgAD mice. (A-C) Confocal microscopy reveals transplanted NSCs (green, GFP) within the alveus of the hippocampus (white matter tract overlying CA1) that secrete high levels of neprilysin (red) three months post-transplantation. (D-F) In contrast, control-transfected NSCs (green, GFP) show no detectable expression of neprilysin (red) in vivo. (G) High magnification of a sNEPNSC-derived neuron shows high levels of sNEP (red) surrounding the engrafted cells. (H) Three months after transplantation, A $B$ plaques (red, OC antibody) within the left side of the hippocampus (SNEP) are significantly reduced versus the contralateral hippocampus (Cntl). No obvious reduction in tau (blue, HT-7 antibody) is observed in aged animals, in line with prior findings that well-established insoluble NFTs are not decreased by Aß-immunotherapy [19]. Scale Bar $=45 \mu \mathrm{m}$ in A-F, $15 \mu \mathrm{m}$ in G, and $160 \mu \mathrm{m}$ in H. A $\beta$, beta-amyloid; NFTs, neurofibrillary tangles; NSCs, neural stem cells; sNEP, secreted neprilysin.

were sacrificed and the brains examined. In the second paradigm, 9- month-old Thy1-APP transgenic mice were transplanted with 100,000 sNEP or control-transduced NSCs unilaterally into the hippocampus. The contralateral hippocampus received an equivalent injection of vehicle. One month after transplantation Thy1-APP mice were sacrificed and the brains examined.

As previously shown [3], GFP-NSCs engraft well into the hippocampus and migrate into the surrounding white matter tracts and overlying cortex (Figures 3A-F and 5G-L). Notably, sNEP-expressing NSCs continue to produce and secrete NEP into the surrounding parenchema for up to three months post-transplantation (Figure 3A-C). In contrast, NEP expression is not detected adjacent to control-transfected NSCs (Figure 3D-F). Confocal imaging reveals NEP surrounding GFP-expressing cell transplants and also diffusing into the adjacent host parenchyma (Figure 3G).

More importantly, when $\mathrm{A} \beta$ levels were assessed, we observed significant reductions in plaque density in areas adjacent to sNEP-NSC grafts (Figure $3 \mathrm{H}$ ). Interestingly, reductions in $\mathrm{A} \beta$ plaque load occurred not only directly adjacent to subiculum grafts, but also within the amygdala and medial septum, two regions that receive substantial inputs directly from the subiculum (Figure 4) [18]. Thus, it appears that sNEP expression may modulate production or transport of $\mathrm{A} \beta$ within efferent axonal projections. Plaque load was also examined in the cingulate and piriform cortices. Although NEP expression tended to reduce $A \beta$ in these two regions, these differences failed to reach 


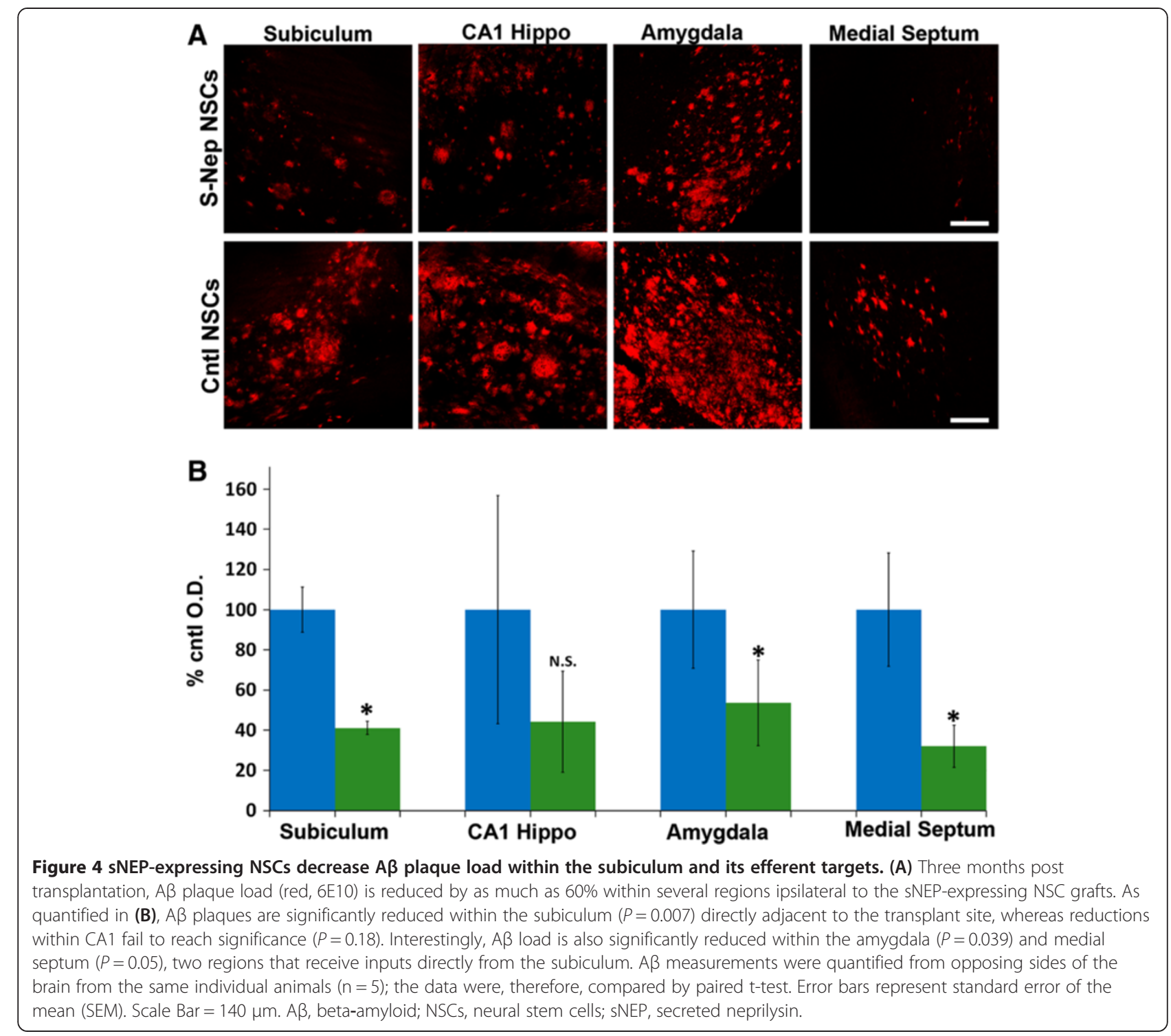

significance $(P=0.27$ and 0.32 , data not shown). A likely explanation for this finding is that cortical plaque load between individual mice varies more than other quantified regions.

\section{Established tangle pathology is unchanged but synaptic density is increased by sNEP-NSCs}

Previous studies have shown that therapies that decrease $\mathrm{A} \beta$ can also reduce tau accumulation and hyperphosphorylation in young 3xTg-AD mice [19]. However, in aged transgenic mice with well-established NFT pathology, A $\beta$-targeted therapies fail to modify tau. To determine whether sNEP-NSCs can alter tau pathology in aged 3xTgAD mice we quantified the levels of AT8 phosphorylated tau (ser199/202) within the hippocampus. Consistent with $A \beta$ vaccination studies, $\mathrm{sNEP}$-expressing NSCs have no effect on well-established tau pathology in 21-month-old
3xTg-AD mice (Figure 5A-C). To determine whether sNEP expression leads to a functional effect on neuronal connectivity, we examined synaptophysin immunoreactivity. As shown, sNEP-NSCs increase synaptic density within the subiculum by $31.8 \%$ versus control-NSCs (Figure $5 \mathrm{D}-\mathrm{E}$, paired t-test, $P=0.009$ ). Thus, sNEP-NSCs provide additional benefit by reducing $\mathrm{A} \beta$-mediated synaptotoxicity. Interestingly, synaptic density was not significantly altered within the medial septum or amygdala (data not shown, $P=0.41$ and 0.54 , respectively). This finding could suggest that the distal effects of NEP expression are somewhat diminished. However, synaptic deficits in these two regions have not yet been described in $3 x \mathrm{Tg}-\mathrm{AD}$ mice; thus, changes in the medial septum and amygdala in response to $\mathrm{A} \beta$ reduction might not be expected.

To determine the differentiated phenotype of engrafted cells, double-labeling for GFP and neuronal and glial 

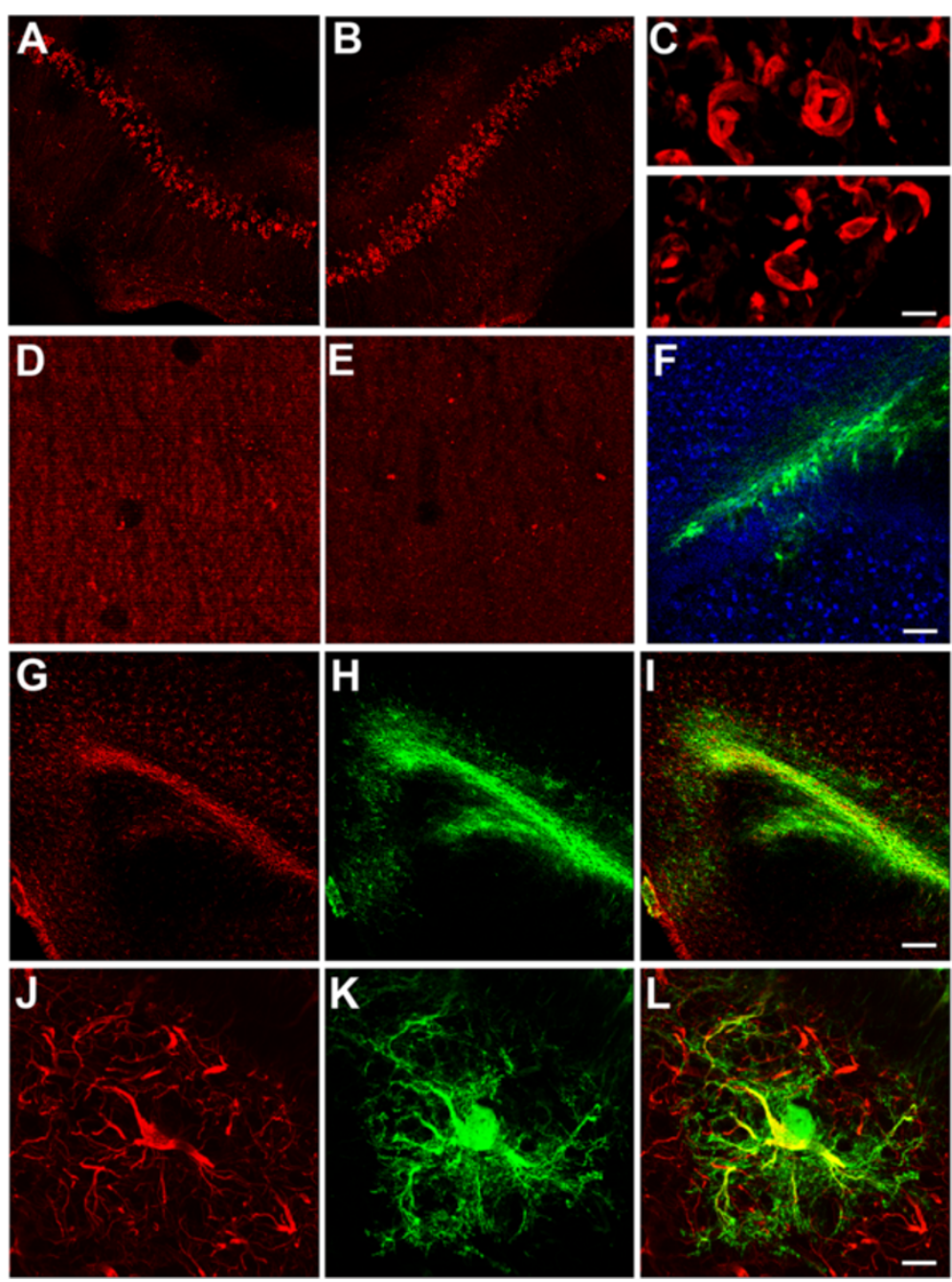

Figure 5 sNEP- NSCs do not alter established tangle pathology but increase synaptic density and differentiate primarily into astrocytes. Aged 3xTg-AD mice develop both AB plaques and neurofibrillary tangles. We, therefore, examined whether sNEP-NSCs modulate tau pathology in vivo. Immunofluorescent labelling and quantification of phosphorylated tau (AT8 epitope: ser199/202) within the hippocampus revealed no significant differences between SNEP-NSC (A, C-top panel) and control-NSC (B, C-bottom panel) treated sides $(n=5)$. This finding is in line with previous reports that decreasing $A \beta$ in aged $3 \times \mathrm{Tg}-\mathrm{AD}$ mice does not reduce established insoluble tangle pathology. To determine whether sNEP expression leads to a functional effect on neuronal connectivity, we examined synaptophysin immunoreactivity. As shown, sNEP-NSCS increase synaptic density within the subiculum (D) by more than $31 \%$ versus control-NSCs ( $\mathbf{E}, P=0.009$, paired t-test). To examine the differentiation of transplanted NSCs, double-labelling for neuronal and glial markers was performed. In agreement with previous studies, very few NSCs (green) transplanted into the aged brain co-express the neuronal marker NeuN (blue, F). In contrast, the great majority of NSCS co-express the astrocytic marker GFAP (red, G-L). Scale Bar $=100 \mu \mathrm{m}$ in A-B, $15 \mu \mathrm{m}$ in C, $6 \mu \mathrm{m}$ in D-E, $15 \mu \mathrm{m}$ in F, $300 \mu \mathrm{m}$ in G-I, and $15 \mu \mathrm{m}$ in J-L. A beta-amyloid; GFAP, glial fibrillary acidic protein; NSCs, neural stem cells; sNEP, secreted neprilysin.

markers was performed. We previously showed that murine NSCs transplanted into aged brains primarily express astrocytic markers $[3,20]$. In this study we found similar results, detecting very few NSCs that co-expressed the neuronal marker NeuN (Figure 5F), but large numbers of GFP-expressing cells that co-expressed the astrocyte marker GFAP in both sNEP and control-NSC engrafted sides (Figure 5G-L). Similar gliogenic differentiation profiles were observed in both 3xTg-AD (Figure 5) and Thy1APP mouse models (data not shown).
Neprilysin-expressing NSCs reduce plaques and increase synaptic density in a second transgenic model of $A D$

Many transgenic models of AD have been generated and conflicting results have occasionally been observed between different models. We, therefore, tested whether sNEP-expressing NSCs can modify A $\beta$-levels in a second independent transgenic AD line. For these experiments, sNEP-expressing NSCs were also independently generated and characterized (Figure 2), providing additional evidence regarding the reproducibility of this approach. 
Thy1-APP mice develop A $\beta$-plaques beginning at three months of age. To examine the effects of NSCs on established pathology, cells were transplanted into ninemonth-old Thy1-APP mice and pathology was examined one month later. As shown, sNEP-expressing NSCs continue to produce NEP one month after transplantation (Figure 6A-E). More importantly, $A \beta$ plaque load is significantly decreased within the ipsilateral hippocampus of sNEP-NSC transplanted mice (Figure 6F-J). In contrast, control-NSCs have no effect on $\mathrm{A} \beta$ pathology (Figure 6I,J). Interestingly, sNEP-expression also reduced the activation of caspase- 3 within NSCs, suggesting that NEP production likely protects engrafted NSCs from $\mathrm{A} \beta$-induced toxicity (Figure 6K-S). To further examine the potential functional effects of sNEP-expression we examined synaptophysin immunoreactivity in Thy1-APP mice. As in 3xTg-AD mice, we again found that sNEPNSCs significantly increase synaptic density (Figure 6T-X).

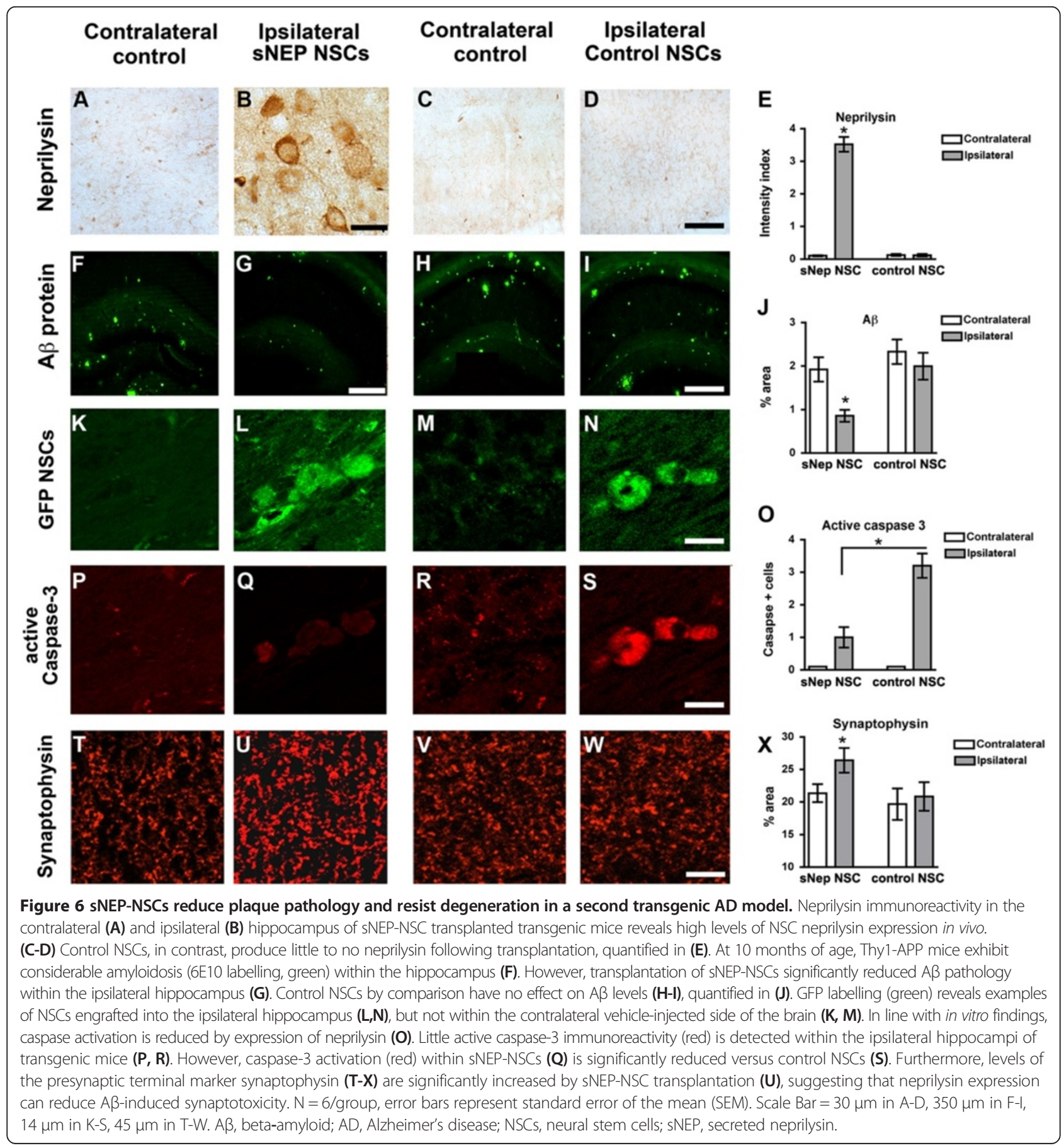


Thus, sNEP-expressing NSCs reduce $A \beta$ pathology and improve synaptic connectivity in two independent transgenic models of $\mathrm{AD}$.

\section{Neprilysin expression may enhance microglial $A \beta$ phagocytosis}

To determine whether NEP expression influences microglial activation state we next examined the microglial marker IBA-1. As one might expect, microglial number was increased adjacent to NSC grafts versus vehicleinjection sites in Thy1-APP mice (Figure 7A,B). Surprisingly, sNEP expression also further elevated microglial number versus control-NSC transplantation. To determine whether this increase in microglial number might further facilitate the degradation of $A \beta$, we next examined the co-localization of microglia and $A \beta$. As shown, we found that NSC NEP expression significantly increased the amount of $A \beta$ detected within adjacent microglia
(Figure 7C,D). Thus, it appears that NEP expression not only directly degrades $A \beta$, but may also enhance the microglial degradation of $A \beta$ and/or $A \beta$ fragments in Thy1-APP mice. In contrast to these findings, we found no significant changes in microglial number in response to NEP in 3xTg-AD mice. A likely explanation for this difference is that the Thy1-APP paradigm examined brains after one month duration whereas the 3xTg-AD study investigated the effects of three-months NSC engraftment. It is, therefore, likely that the microglial activation state has significantly diminished by three months post transplantation.

\section{Discussion}

NSC transplantation may provide a promising new approach to treat $\mathrm{AD}$ by elevating neurotrophin levels and enhancing endogenous synaptic connectivity [3]. However, NSCs do not modify $A \beta$ pathology; thus, the long-

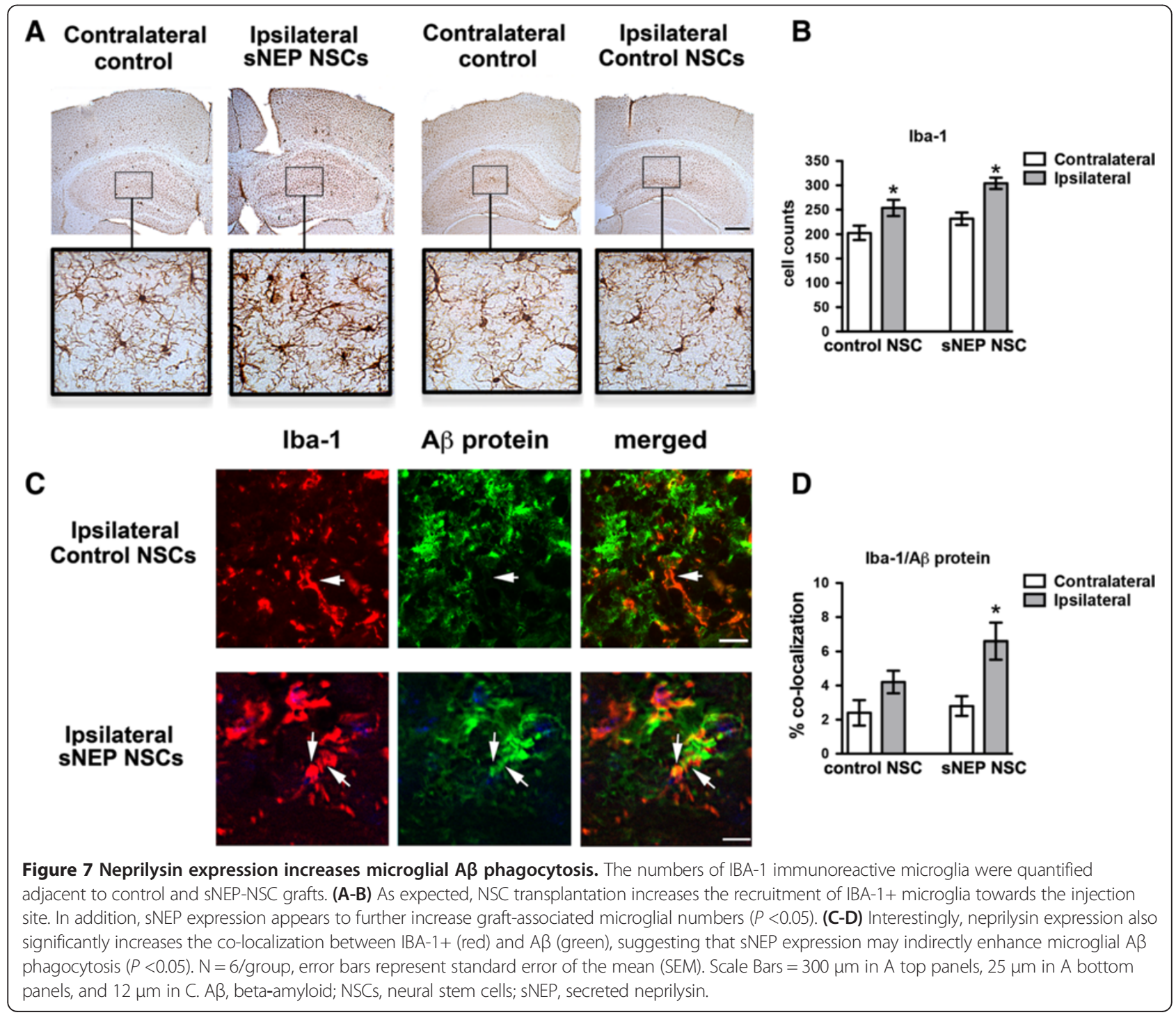


term benefit of NSC transplantation remains unclear. Because of their robust migratory capacity, NSCs provide a compelling approach to deliver therapeutic proteins to the brain. By combining the inherent benefits of NSC transplantation with ex vivo gene therapy, modified NSCs may provide a powerful combinatorial approach to treat $\mathrm{AD}$. In this study, we examined whether NEP-expressing NSCs could target and reduce A $\beta$ pathology in two well-established transgenic models of AD. We found that NSCs could be readily modified to express and secrete NEP without altering multipotentcy or differentiation potential. More importantly, sNEPexpressing NSCs dramatically reduced A $\beta$ levels both in vitro and in vivo and elevated synaptic density in both transgenic models of AD.

We and others previously tested the effect of NEP overexpression in transgenic $\mathrm{AD}$ mice $[7,10,21,22]$. Each of these studies observed significant reductions in $A \beta$ plaque load in response to NEP. We also previously demonstrated improved cognitive function in $\mathrm{AD}$ mice following lentiviral delivery of sNEP [21]. In contrast, Meilandt and colleagues found no improvement in cognition when hAPP-J20 mice were crossed to mice overexpressing membrane-bound NEP [22]. Major differences in delivery approach and the use of secreted versus membrane-bound forms of NEP likely account for these differences. In the current study, we again utilized the secreted form of neprilysinNEP, but instead used NSCs as a delivery vehicle. Potential benefits of NSC-mediated delivery over viral methods include a greater distribution of NEP delivery. Whereas NSCs have previously been shown to migrate through the brain parenchema [12], viral-based approaches typically provide only a small radius (approximately $0.5 \mathrm{~mm}$ ) of infectivity [11]. As the human brain is approximately three thousand times larger than the mouse brain, scale-up of viral gene-therapy for clinical translation remains a considerable challenge. Peripheral delivery of NEP protein also appears to be ineffective, as a recent study found that intravenous delivery of a NEP fusion protein could reduce plasma $A \beta$, but failed to clear $A \beta$ plaques within the brain [23]. New approaches, such as stem cell mediated delivery, may therefore be needed to provide broader expression of therapeutic proteins in the brain. However, it remains to be determined whether the migratory capacity of NSCs would be sufficient to treat a widespread brain disease such as AD. Clearly NSCbased delivery provides a benefit over current gene therapy approaches, but multiple NSC injections would likely still be needed.

The unilateral transplantation design used in this study allowed us to directly compare plaque load and synaptic density within the same animals. Unfortunately, this design also precluded further biochemical analysis of soluble $A \beta$. It, therefore, remains possible that soluble $A \beta$ levels are not altered by this approach. However, the observed sNEP-NSC mediated a $31.8 \%$ increase in synaptic density, suggesting that soluble $A \beta$ oligomers are also likely reduced. Previous studies also support this notion, as lentiviral-mediated delivery of s-NEP reduces both soluble and insoluble $A \beta$ [21]. As most transgenic AD models, including those used, exhibit little or no neuronal loss we conclude that the effect of sNEP-NSCs on synaptic density is mediated via maintenance and/or enhancement of endogenous synaptic connectivity. It is important to note that synapse loss correlates strongly with cognitive dysfunction in AD patients [24]. Quite notably in the current study we observed a $31.8 \%$ increase in hippocampal synaptic density three months after sNEP-NSC transplantation. The magnitude of this effect is similar to the approximately $38 \%$ loss of synapses that occurs in AD patients [24]. We, therefore, conclude that the effect of sNEP-NSCs on synaptic density represents a meaningful functional outcome. In addition to $A \beta$ plaques and synaptic loss, cerebral amyloid angiopathy (CAA) represents another important $\mathrm{AD}$-associated pathology. However, neither the 3xTg-AD nor Thy1-APP models develop CAA at the ages studied. Future experiments are therefore needed to determine whether NSCmediated NEP delivery can also influence CAA.

Other cell types may also be useful for delivering NEP to the AD brain. For example, induced pluripotent stem cells (iPSCs) offer an alternative and extremely promising new cell source that could be used to deliver NEP and personalized cell therapies. Two distinct advantages of iPSC-derived NSCs over allogeneic fetal-derived NSCs include the greatly increased capacity for scale-up and the potential ability to transplant a patient's own cells, thereby reducing or eliminating the need for immunesuppression. Most recently, xeno-free clinical grade iPSCs have been generated, moving this approach several important steps closer to clinical reality [25].

Peripheral-derived cells are also worth considering. In support of this, Lebson and colleagues transfected CD11b + monocytes with NEP and infused these cells biweekly into AD transgenic mice [26]. Some of these modified monocytes migrated into the brain and $A \beta$ deposition was slowed. This promising approach further supports the notion that cell-based delivery of NEP can be used to target $A \beta$. However, the use of monocytes offers both advantages and disadvantages in comparison to NSC-based delivery. Monocytes, for example, have limited half-lives; thus, repeated injections are required. This potential drawback can also be viewed as a possible advantage by providing some protection against adverse events. Interestingly, upregulation of NEP, also known as CALLA (common acute lymphoblastic leukemia antigen), commonly occurs in acute leukemias [27]. The 
overexpression of NEP in hematopoetic lineages is therefore concerning. It is also worth noting that without irradiation, concurrent stroke, or additional treatments, very few monocytes appear to migrate into the $A D$ brain [28]. Thus, monocyte mediated delivery of NEP may not provide as robust a therapeutic approach as NSC-based delivery.

In contrast, our data suggest that the disease-modifying effects of sNEP-NSCs can be extremely robust, modulating $A \beta$ plaques in regions both adjacent to and interconnected with the grafted area. Reductions in local $A \beta$ appear to occur via both NEP-mediated proteolysis and enhanced microglial degradation. The mechanism(s) by which SNEP-NSCs decrease plaque load in more distant regions, such as the amygdala and medial septum, however, remains unclear. Transplanted NSCs did not migrate into the amygdala or medial septum and we were unable to detect clear evidence of axonally-transported NEP. sNEP-NSCs may instead modulate the production and/or anterograde transport of $\mathrm{A} \beta$ from the hippocampus to these efferent targets. Intriguingly, recent studies point toward a network diffusion model for the transmission of neurodegenerative pathologies via synaptic networks $[29,30]$. In light of this, our results could indicate that therapeutic proteins might also effectively modulate pathology via the same neural networks.

\section{Conclusions}

In conclusion, the current study clearly demonstrates that sNEP-expressing NSCs offer a novel and highly effective combinatorial approach to modify AD pathology and enhance synaptic connectivity. Future studies will be needed to determine whether this combinatorial approach can also provide additional long-term behavioral efficacy and whether such approaches can be translated towards an eventual clinical application.

\section{Abbreviations \\ 3xTg-AD: triple transgenic mouse model of Alzheimer's Disease AD: Alzheimer's Disease; ANOVA: analysis of variance; BDNF: brain-derived neurotrophic factor; A : beta-amyloid; CALLA: common acute lymphoblastic leukemia antigen; (D)MEM: (Dulbecco's) modified Eagle's medium; EGF: epidermal growth factor; ELISA: enzyme-linked immunosorbent assay; GFAP: glial fibrillary acid protein; GFP: green fluorescent protein; LDH: lactate dehydrogenase; MHC: major histocompatibility complex; NEP: neprilysin; NFTs: neurofibrillary tangles; NSC: neural stem cell; PBS: phosphate-buffered saline; PLSD: protected least significant difference; ROI: region of interest; sNEP: secreted neprilysin; sox2: sex determining region Y-box 2; \\ Thy1: thymus cell antigen 1.}

\section{Competing interests}

The authors declare they have no competing interests.

\section{Authors' contributions}

MBJ: conception and design, 3xTg-AD transplantation studies, data collection and analysis, financial support, manuscript writing and final approval of the manuscript. BS: generation of neprilysin constructs and viruses, data collection and analysis, final approval of the manuscript. SM: Thy1-APP transplantation studies, data collection, and analysis, final approval of the manuscript. NAC: 3xTg-AD transplantation studies, data collection and analysis, final approval of the manuscript. AAA: 3xTg-AD transplantation studies, data collection and analysis, and final approval of the manuscript. JLD: 3xTg-AD transplantation studies, data collection and analysis, and final approval of the manuscript. FJM: conception and design, manuscript writing and final approval of the manuscript. JFL: conception and design, manuscript writing and final approval of the manuscript. EM: conception and design, data collection and analysis, financial support, manuscript writing and final approval of the manuscript. FML: manuscript writing, financial support, and final approval of the manuscript. All authors read and approved the final manuscript.

\section{Acknowledgements}

This work was supported by CIRM TR1-01245 and the American Health Assistance Foundation (FML), AG029378, AG16573 and Alzheimer's Association NIRG 12-242-550 (MBJ), AG022074, AG18440, AG10435 (EM), the Else-Kröner Fresenius Stiftung (FJM), and CIRM RT1-01108 and Alzheimer's Association IIRG-05-14975 (JFL).

\section{Author details}

${ }^{1}$ Department of Neurobiology and Behavior and Institute for Memory Impairment and Neurological Disorders, University of California Irvine, Irvine, CA 92697, USA. ${ }^{2}$ Center for Regenerative Medicine, the Scripps Research Institute, La Jolla, CA 92037, USA. ${ }^{3}$ Center for Psychiatry (ZIP Kiel), University Hospital Schleswig Holstein, Kiel 24105, Germany. ${ }^{4}$ Department of Neurosciences, University of California San Diego, La Jolla, CA 92093, USA.

Received: 4 November 2013 Revised: 14 January 2014

Accepted: 18 February 2014 Published: 16 Apr 2014

\section{References}

1. Thies W, Bleiler L: Alzheimer's disease facts and figures. Alzheimers Dement 2011, 2011:208-244.

2. Prince MJ, Ferri CP, Sousa R, Albanese E, Ribeiro WS, Honyashiki M: World Alzheimer Report 2009: The Global Prevalence of Dementia. London: Alzheimer Disease International; 2009. Available at: http://www.alz.co.uk/ research/files/WorldAlzheimerReport.pdf.

3. Blurton-Jones M, Kitazawa M, Martinez-Coria H, Castello NA, Muller FJ, Loring JF, Yamasaki TR, Poon WW, Green KN, LaFerla FM: Neural stem cells improve cognition via BDNF in a transgenic model of Alzheimer disease. Proc Natl Acad Sci U S A 2009, 106:13594-13599.

4. Hampton DW, Webber DJ, Bilican B, Goedert M, Spillantini MG, Chandran S: Cell-mediated neuroprotection in a mouse model of human tauopathy. J Neurosci 2010, 30:9973-9983.

5. Lee HJ, Lee JK, Lee H, Carter JE, Chang JW, Oh W, Yang YS, Suh JG, Lee BH, Jin HK, Bae JS: Human umbilical cord blood-derived mesenchymal stem cells improve neuropathology and cognitive impairment in an Alzheimer's disease mouse model through modulation of neuroinflammation. Neurobiol Aging 2010, 33:588-602.

6. Iwata N, Tsubuki S, Takaki Y, Watanabe K, Sekiguchi M, Hosoki E, Kawashima-Morishima M, Lee HJ, Hama E, Sekine-Aizawa Y, Saido TC: Identification of the major Abeta1-42-degrading catabolic pathway in brain parenchyma: suppression leads to biochemical and pathological deposition. Nat Med 2000, 6:143-150.

7. Leissring MA, Farris W, Chang AY, Walsh DM, Wu X, Sun X, Frosch MP Selkoe DJ: Enhanced proteolysis of beta-amyloid in APP transgenic mice prevents plaque formation, secondary pathology, and premature death. Neuron 2003, 40:1087-1093.

8. Caccamo A, Oddo S, Sugarman MC, Akbari Y, LaFerla FM: Age- and region-dependent alterations in Abeta-degrading enzymes: implications for Abeta-induced disorders. Neurobiol Aging 2005, 26:645-654.

9. Wang S, Wang R, Chen L, Bennett DA, Dickson DW, Wang DS: Expression and functional profiling of neprilysin, insulin-degrading enzyme, and endothelin-converting enzyme in prospectively studied elderly and Alzheimer's brain. J Neurochem 2010, 115:47-57.

10. Marr RA, Rockenstein E, Mukherjee A, Kindy MS, Hersh LB, Gage FH, Verma IM, Masliah E: Neprilysin gene transfer reduces human amyloid pathology in transgenic mice. J Neurosci 2003, 23:1992-1996.

11. de Backer MW, Brans MA, Luijendijk MC, Garner KM, Adan RA: Optimization of adeno-associated viral vector-mediated gene delivery to the hypothalamus. Hum Gene Ther 2010, 21:673-682.

12. Muller FJ, Snyder EY, Loring JF: Gene therapy: can neural stem cells deliver? Nat Rev Neurosci 2006, 7:75-84. 
13. Billings LM, Oddo S, Green KN, McGaugh JL, LaFerla FM: Intraneuronal Abeta causes the onset of early Alzheimer's disease-related cognitive deficits in transgenic mice. Neuron 2005, 45:675-688.

14. Oddo S, Caccamo A, Shepherd JD, Murphy MP, Golde TE, Kayed R, Metherate R, Mattson MP, Akbari Y, LaFerla FM: Triple-transgenic model of Alzheimer's disease with plaques and tangles: intracellular Abeta and synaptic dysfunction. Neuron 2003, 39:409-421.

15. Rockenstein E, Mallory M, Mante M, Sisk A, Masliaha E: Early formation of mature amyloid-beta protein deposits in a mutant APP transgenic model depends on levels of Abeta(1-42). J Neurosci Res 2001, 66:573-582.

16. Mizumoto H, Mizumoto K, Shatos MA, Klassen H, Young MJ: Retinal transplantation of neural progenitor cells derived from the brain of GFP transgenic mice. Vision Res 2003, 43:1699-1708.

17. Blurton-Jones M, Laferla FM: Pathways by which Abeta facilitates tau pathology. Curr Alzheimer Res 2006, 3:437-448.

18. Pitkanen A, Pikkarainen $M$, Nurminen $N$, Ylinen A: Reciprocal connections between the amygdala and the hippocampal formation, perirhinal cortex, and postrhinal cortex in rat. A review. Ann N Y Acad Sci 2000, 911:369-391.

19. Oddo S, Billings L, Kesslak JP, Cribbs DH, LaFerla FM: Abeta immunotherapy leads to clearance of early, but not late, hyperphosphorylated tau aggregates via the proteasome. Neuron 2004, 43:321-332.

20. Yamasaki TR, Blurton-Jones M, Morrissette DA, Kitazawa M, Oddo S, LaFerla FM: Neural stem cells improve memory in an inducible mouse model of neuronal loss. J Neurosci 2007, 27:11925-11933.

21. Spencer B, Marr RA, Rockenstein E, Crews L, Adame A, Potkar R, Patrick C, Gage FH, Verma IM, Masliah E: Long-term neprilysin gene transfer is associated with reduced levels of intracellular Abeta and behavioral improvement in APP transgenic mice. BMC Neurosci 2008, 9:109.

22. Meilandt WJ, Cisse M, Ho K, Wu T, Esposito LA, Scearce-Levie K, Cheng IH, Yu GQ, Mucke L: Neprilysin overexpression inhibits plaque formation but fails to reduce pathogenic Abeta oligomers and associated cognitive deficits in human amyloid precursor protein transgenic mice. J Neurosci 2009, 29:1977-1986.

23. Walker JR, Pacoma R, Watson J, Ou W, Alves J, Mason DE, Peters EC, Urbina HD, Welzel G, Althage A, Liu B, Tuntland T, Jacobson LH, Harris JL, Schumacher AM: Enhanced proteolytic clearance of plasma abeta by peripherally administered neprilysin does not result in reduced levels of brain abeta in mice. J Neurosci 2013, 33:2457-2464.

24. Terry RD, Masliah E, Salmon DP, Butters N, DeTeresa R, Hill R, Hansen LA, Katzman R: Physical basis of cognitive alterations in Alzheimer's disease: synapse loss is the major correlate of cognitive impairment. Ann Neurol 1991, 30:572-580.

25. Awe JP, Lee PC, Ramathal C, Vega-Crespo A, Durruthy-Durruthy J, Cooper A, Karumbayaram S, Lowry WE, Clark AT, Zack JA, Sebastiano V, Kohn DB, Pyle AD, Martin MG, Lipshutz GS, Phelps PE, Pera RA, Byrne JA: Generation and characterization of transgene-free human induced pluripotent stem cells and conversion to putative clinical-grade status. Stem Cell Res Ther 2013, 4:87.

26. Lebson L, Nash K, Kamath S, Herber D, Carty N, Lee DC, Li Q, Szekeres K, Jinwal U, Koren J, Dickey CA, Gottschall PE, Morgan D, Gordon MN: Trafficking CD11b-positive blood cells deliver therapeutic genes to the brain of amyloid-depositing transgenic mice. J Neurosci 2010, 30:9651-9658.

27. Bene MC, Faure GC: CD10 in acute leukemias. GEIL (Groupe d'Etude Immunologique des Leucemies). Haematologica 1997, 82:205-210.

28. Rezai-Zadeh K, Gate D, Gowing G, Town T: How to get from here to there: macrophage recruitment in Alzheimer's disease. Curr Alzheimer Res 2011, 8:156-163.

29. Palop JJ, Mucke L: Amyloid-beta-induced neuronal dysfunction in Alzheimer's disease: from synapses toward neural networks. Nat Neurosci 2010, 13:812-818.

30. Raj A, Kuceyeski A, Weiner M: A network diffusion model of disease progression in dementia. Neuron 2012, 73:1204-1215.

\section{$10.1186 /$ scrt440}

Cite this article as: Blurton-Jones et al:: Neural stem cells geneticallymodified to express neprilysin reduce pathology in Alzheimer transgenic models. Stem Cell Research \& Therapy 2014, 5:46

\section{Submit your next manuscript to BioMed Central and take full advantage of:}

- Convenient online submission

- Thorough peer review

- No space constraints or color figure charges

- Immediate publication on acceptance

- Inclusion in PubMed, CAS, Scopus and Google Scholar

- Research which is freely available for redistribution

Submit your manuscript at www.biomedcentral.com/submit
Biomed Central 